\title{
Multi-Objective Distribution Network Expansion Incorporating Electric Vehicle Charging Stations
}

\author{
Yue Xiang ${ }^{1, *}$, Wei Yang ${ }^{1}$, Junyong $\mathrm{Liu}^{1}$ and Furong $\mathrm{Li}^{2}$ \\ 1 School of Electrical Engineering and Information, Sichuan University, Chengdu 610065, China; \\ yangwei_scu@126.com (W.Y.); liujy@scu.edu.cn (J.L.) \\ 2 Department of Electronic and Electrical Engineering, University of Bath, Bath BA2 7AY, UK; f.li@bath.ac.uk \\ * Correspondence: exxyye@gmail.com; Tel.: +86-139-8047-2431
}

Academic Editor: Chunhua Liu

Received: 31 August 2016; Accepted: 27 October 2016; Published: 3 November 2016

\begin{abstract}
The paper develops a multi-objective planning framework for distribution network expansion with electric vehicle charging stations. Charging loads are modeled in the first place, and then integrated into the optimal distribution network expansion planning. The formulation is extended from the single objective of the economic cost minimization into three objectives with the additional maximization of the charging station utilization, and maximization of the reliability level. Compared with the existing models, it captures the interactive impacts between charging infrastructures planning and distribution network planning from the aspects of economy, utilization, and reliability. A multi-stage search strategy is designed to solve the multi-objective problem. The models and the strategy are demonstrated by the test case. The results show that the proposed planning framework can make a trade-off among the three objectives, and offer a perspective to effectively integrate the network constraints from both the transportation network and distribution network.
\end{abstract}

Keywords: charging station; distribution network expansion planning; multi-objectives; multi-stage search strategy; traffic flow

\section{Introduction}

Driven by low-carbon targets and the development of the smart city, the growing number of electric vehicles (EVs) is becoming a matter of concern not only in the transportation system but also in the smart grid [1,2]. The integration of EVs into the distribution network with an uncertain pattern increases peak loading on feeders, power loss, voltage deviations, and the need for network reinforcements [3-5]. In addition, large-scale adoption of EVs would also have a significant impact on the transportation network [6]. The availability of EV charging infrastructures is a crucial factor in increasing the adoption of EVs in the transportation and distribution system. Therefore, it is important to understand how distribution planning can be adjusted when EV charging loads penetrate into the distribution system; it will also be necessary to adjust the charging infrastructure plan to suit such penetration not only at the distribution but also at the transportation level.

Charging stations (CSs) should be placed at appropriate locations and be scaled correctly such that an EV can easily access a CS within its driving range. Several studies have already been carried out to deploy CSs. Inspired by the base station towers' placement to communicate with all mobile nodes, an allocation approach was proposed for deploying the CSs in [7]. A Voronoi diagram was introduced and integrated with the economic planning model for CSs placement in [8]. In [9], the criteria performance of different alternatives and criteria weights were proposed and judged by five groups of expert panels in the environmental, economic, and social criteria associated with a total of sub-criteria, which could help make an assessment of the performance of the system with the charging load from the CSs in 
the corresponding deployment. In [10], a two-step screening method considering the environmental factors and the service radius of EV charging stations was presented to identify the optimal sites of EV charging stations, and then a mathematical model for the optimal sizing of EV charging stations was developed with the minimization of total cost associated with EV charging stations. In [11], based on the concept of set covering and vehicle refueling logics, a model with dual objectives of maximum coverage and minimum cost was proposed to determine the number and locations of fast-refueling stations simultaneously serving intercity and intra-city travel. In [12], with the aim of maximizing the number of vehicles served while staying within the budget constraints, a mathematical programming model was developed to site the refueling stations. Joana Cavadas et al. proposed an improved model for locating EV charging stations [13]. This model could avoid double counting the demand by taking the successive activities of the travellers into account. Considering an EV charging network equipped with different charging technologies, two frameworks were proposed in [14]: for large networks, the first framework leveraged pricing dynamics in order to control the EV customer request rates and to provide a charging service with the best level of quality of service (QoS), and for smaller networks, the objective was to compute the minimum amount of resources required to provide certain levels of QoS to each class.

Considering transportation effects on siting CSs, flow-capturing locational model (FCLM) was investigated in [15]. All trips from the same origin-destination (OD) pair of traffic flow were assigned to several paths, in which the site of CSs could be selected according to the travel habits or behaviors. Therefore, the proposed model would choose the minimum number of CSs in areas that had high levels of traffic flow. However, in that study, the effects on the distribution system were not considered, so the deployment of CSs may not be fit for the capability of the corresponding distribution system. Considering the above two aspects, optimal planning models for CS planning in the distribution network were proposed and studied in [16]. Furthermore, load template profiles were introduced to verify the CS planning result based on an integrated distribution and transportation constrained model, which was presented in [17]. Considering the power distribution and traffic network topologies and the EV owner's driving behavior, a new multi-objective charging station planning method was formulated in [18] to ensure charging service while reducing power losses and voltage deviations of distribution systems.

It is evident that the impact of EV charging on the distribution system could be significant as penetration increases in the coming years. So, EV load demand needs to be included in the distribution network expansion planning process. According to previous related studies, optimal distribution network expansion planning (ODNEP) is formulated as a mixed-integer nonlinear programming (MINLP) optimization problem, and used to determine the optimal site and size of feeders and substations [19]. For example, considering the dynamic behaviours of the system parameters asset management and geographical constraints, the developed imperialist competitive algorithm was employed for the proposed multistage expansion planning in [20]. Integrated with EV charging load, the siting and sizing of CSs would also be integrated into the distribution network planning model. It was indicated from [21] that ODNEP with optimal siting and sizing of CSs could effectively reduce investment and operation costs. Hu et al. proposed a mathematical model for distribution network expansion planning, taking optimal siting and sizing of EV charging station into account [22]. Additionally, in the proposed model, the existing substation could be expanded, and the feeders could be replaced or newly built. Integrated with the vehicle to grid (V2G) strategy, the coordinated planning problem was also investigated in [23]. Additionally, a multi-objective formula was proposed in [24] to determine the optimal EV penetration, generation units and feeder upgrades. The above studies discussed the integrated planning of distribution network and CSs from several aspects. However, the diversity of travel patterns and traffic flow aspects is not considered, which may lead to locating CSs or the other distribution components at sites with a certain size favorable for electrical utilities but not easy for drivers to access due to not including traffic flow aspects. 
The problem we need to solve in this study is that without detailed knowledge about EV travel range, drivers' travel habits, and accessibility at this area, it is difficult to a reasonable distribution network expansion plan. Using the OD data from transportation departments and existing power related data from electricity utility at hand, we must try to suit both power and charging demands. Thus, the aim of this work is to present an integrated distribution network expansion planning framework that takes into account the EV charging loads and network constraints from both the distribution and transportation systems. The main contributions are listed as follows:

- A comprehensive distribution network expansion planning framework is proposed to determine the optimal size, site, and period of operation of substations, feeders, and CSs.

- Multi-objectives are designed in the optimal model, in which the minimization of long-run and short-run costs, the maximum of CS utilization, and the maximum of reliability are formulated from three perspectives.

- A novel set of transportation and distribution operational constraints are included in the planning framework to capture EV travel behaviour on the transportation network and charging effects on the distribution network.

- A multi-stage search strategy is designed for the multi-objective optimization problem considering the network constraints of the two geographical coupled systems.

The presented model will be a useful tool not only for planning purposes, but also for evaluating the existing EV charging integrated network from the transportation and distribution perspectives.

Therefore, a brief organization and implementation of the integrated expansion planning framework in the paper is presented as shown in Figure 1.

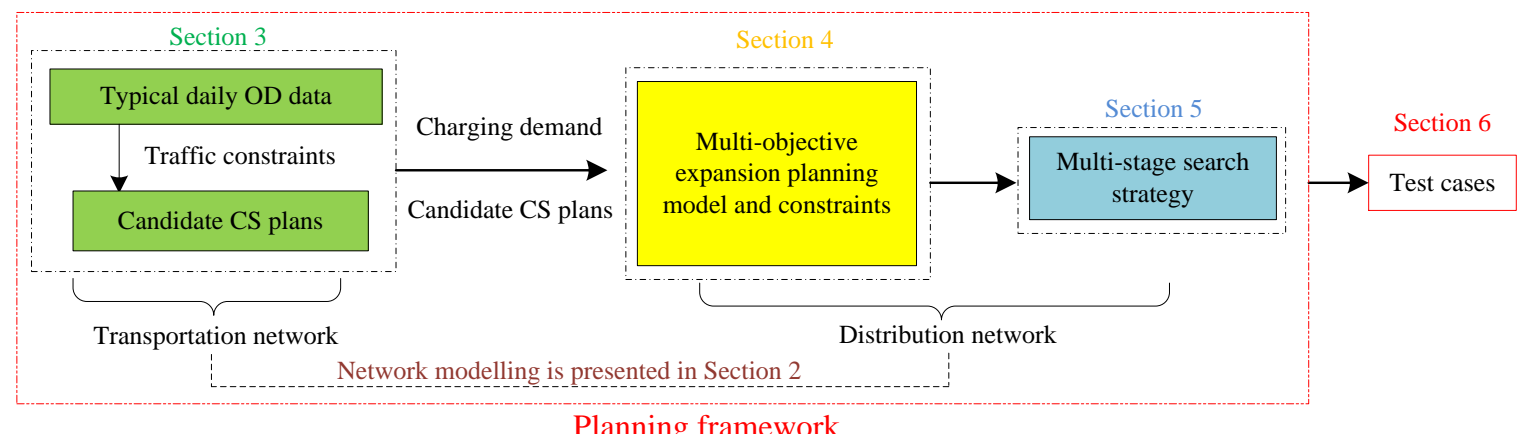

Planning framework

Figure 1. Integrated expansion planning framework.

As depicted in Figure 1, the integrated expansion planning framework mainly includes three parts. Firstly, the typical daily OD data are optimally assigned on the transportation system considering traffic constraints based on our previous research [17], which are then used in the queuing model to determine the candidate CS plans at the transportation network. In that way, the fast charging demands of each CS are calculated at the corresponding distribution network, while the slow charging demands of each bus can be obtained based on the conventional load and charging pattern statistics data. Then, a multi-objective distribution network expansion planning model is developed, which considers the cost, the CS utilization level, and the reliability level with the integration of EVs. Furthermore, a multi-stage search strategy is designed for solving the proposed model, including the improved topology generation algorithm for distribution network with several substations and a bargaining function-based multi-objective decision method.

The rest of the paper is organized as follows. In Section 2, the network modeling is introduced, as well as the basic data needed to prepare for the planning. The flowchart for determining the capacity of the CSs in a candidate plan and the corresponding charging loads for planning are described in Section 3. Then, a constrained multi-objective model, including objectives of the economic cost, 
CS utilization, and reliability level, is proposed in Section 4. A multi-stage search strategy for solving the planning problem is given in Section 5. A test case is illustrated in Section 6 and conclusions are given in Section 7.

\section{Network Modeling}

The EV charging infrastructures mainly include charging piles (CPs) and charging stations (CSs). For simplicity, it is assumed that CPs for slow charging exist in residential and commercial areas with conventional load. The fast charging is handled by the CSs whose siting and sizing are optimized in the paper. The traffic flow could indicate the aggregated intensity of the EVs to the particular location, which can be used as a reference for the charging infrastructure planning. Furthermore, the charging demand of EVs would bring additional loads to the distribution network. Thus, the planning for CSs should be integrated both with the distribution network (D-network) and the transportation network (T-network), as shown in Figure 2. Let GD (ND, LD) denote the D-network, where ND and LD are the sets of buses and lines, respectively. GT (NT, LT) denotes the T-network in the same urban area that shares geographic information with GD, where NT and LT are the sets of nodes and links, respectively. Assuming that travel demands originate from a set of origin nodes, and are destined for a set of destinations $S \in N_{\mathrm{T}}$, which are defined as OD pairs, CSs are to be planned at the nodes of the T-network and connected to the corresponding buses in the D-network through special transformers and lines. Thus, it can be seen that a certain node in the T-network may overlap with a corresponding bus in the D-network, which is defined as a "node-bus" pair. Without losing generality, it is assumed that there is no more than one CS at each node.

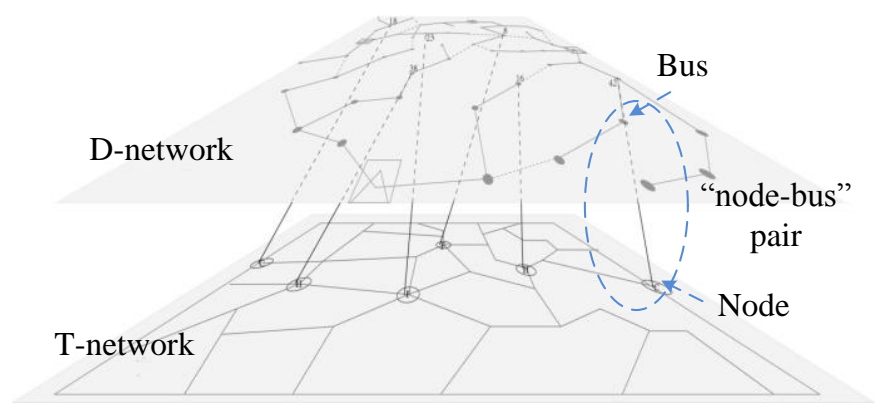

Figure 2. Geographically coupled networks.

The following objectives are going to be included in the distribution network expansion:

- New substation construction or an expansion: A medium-voltage distributed network is considered in this paper, so the size of the new substation should not be too large, and can be in the form of a photovoltaic (PV) based storage substation or distributed generation (DG).

- New line (feeder) construction: It follows the close-loop design and open-loop operation rule. Moreover, the normal open interconnection lines should be determined.

- The siting and size of the CSs: The site is selected from the candidate nodes and the size reflects the number of deployed charging devices.

Therefore, the coordinated expansion planning can be actually implemented on the two networks respectively, i.e., siting and sizing determination for the CSs in the T-network, and the further ODENP with the estimated charging load in the D-network. Before starting to plan, basic data need to be collected, such as the distribution of the residential area and the commercial area with the typical load profile [25], typical parking demand profiles [26], the scale evolution, and daily charging times $\mathrm{H}$, which could be estimated based on multi-factor including the EV scale in the area, the statistical proportion of EVs, the charging frequency per vehicle [27], the typical ratio of fast charging and slow charging (let $\omega(0<\omega<1)$ be the ratio of charging times in CSs in the typical day, while that for CPs would be $(1-\omega))$ [28], and so on. 


\section{Charging Load Modeling}

In this paper, traffic flow data is used to help deploy the CSs and estimate the corresponding charging demand. According to the daily trip OD data obtained from a transportation survey, the traffic flow on each road of the T-network, which is used for planning, can be obtained. The details of the process can be found in [17].

According to the ratio of the traffic flow captured by different candidate nodes in the T-network, we can similarly estimate the ratio of vehicles or the number of EVs that drive pass the node and would have a larger probability of going for a fast charge. Therefore, the approximate average number of EVs arriving at the CS located at node $j$ could be estimated as:

$$
\lambda_{j, t}=H \omega \varepsilon_{t} \frac{f n_{j, t}}{\sum_{j \in \Omega_{\mathrm{T}}} f n_{j, t}} / \Delta t
$$

The mobility and randomness of EVs in a CS are simulated based on the queuing model [29]. Assuming the arrival of EV in a certain CS is described as a Poisson process, the average arrival rate is reflected by $\lambda_{j, t}$ and the average service rate of a charging device is formulated as:

$$
\beta_{j, t}=\frac{\lambda_{j, t}}{s_{j} \mu}
$$

Besides $\lambda_{j, t}$ and $\beta_{j, t}$, other performance indices of the CS service system based on the queuing model can also be calculated as follows:

$$
\begin{gathered}
\rho=\frac{\lambda}{\mu} \\
p_{0}=1 /\left[\sum_{n=0}^{s-1} \frac{\rho^{n}}{n !}+\frac{\rho^{s}}{s !} \frac{1}{1-\beta}\right] \\
W_{q}=\frac{s \rho^{s+1} p_{0}}{\lambda s !(s-\rho)^{2}}
\end{gathered}
$$

In theory, more charging devices require more investment, but it may not be an economical solution. So the minimum number of devices is designed based on the average waiting time $W_{q}$, which is used to indicate customers' patience when charging in the CS. That is to say, if the waiting time exceeds a given criteria, the customer will leave, i.e., $W_{q} \leq W_{q}^{\max }$ is used to determine the minimum number of charging devices. However, it is difficult to get the inverse functions of Equation (5) and obtain the appropriate result directly, thus an enumeration method is used as follows. For the candidate CS at node $j$, according to the maximum $\lambda_{j}$ among time periods, initialize $s$, calculate $W_{q}$, compare it with the given $W_{q}^{\max }$, and let new $s$ equals to $(s+1)$ till $W_{q} \leq W_{q}^{\max }$. Then the corresponding $s$ would be the cost-effective number of charging devices.

After the number of charging devices in a candidate CS plan is obtained, the charging load of the $\mathrm{CS}$ at bus $j$ can be estimated as:

$$
P_{j, t}^{\mathrm{CS}}=\beta_{j, t} s_{j} P_{\mathrm{CD}} \omega
$$

Regarding CPs, assuming they are aggregated at each load bus, the number of CPs at bus $i$ can be similarly estimated according to the load demand ratio and the fast charging times, as shown in Equation (7). Then the equivalent aggregated charging load from the CP at bus $i$, as shown in Equation (8), can be added into the final load demand of bus $i$ in the time period $t$ for the coordinated planning in the next step.

$$
N_{i}^{\mathrm{CP}}=\left\lceil\frac{H \times(1-\omega) \times \sum_{t=1}^{T} P_{\mathrm{L} i, t}}{\kappa \times(1-\gamma) \times \sum_{i=1}^{N_{\mathrm{D}}} \sum_{t=1}^{T} P_{\mathrm{L} i, t}}\right\rceil
$$




$$
P_{i, t}^{\mathrm{CP}}=P_{\mathrm{CP}} \chi_{i, t} N_{i}^{\mathrm{CP}}
$$

where \lceil\rceil is the round up function and $\chi_{i, t}$ is quantified by the normalized parking demand coefficient, which can be obtained by surveys at the corresponding residential or business area of bus $i$ in time period $t$.

\section{Multi-Objective Planning Modeling}

After the candidate plans for CSs and the corresponding charging load are obtained, a multi-objective model for ODNEP is built. It captures the features from both the T-network and D-network, and can achieve a balanced trade-off between three objectives, which are made up as shown in Figure 3.

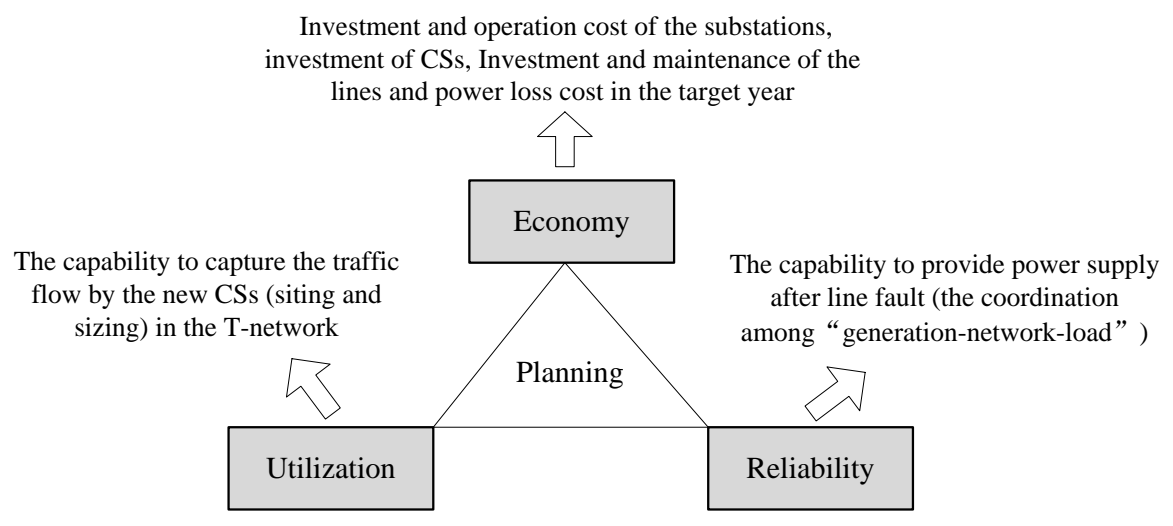

Figure 3. Three objectives for planning.

The economy of the plan is reflected by $F_{C}$, which is the total cost in the target year; the utilization is represented by the average annual captured traffic flow $F_{T}$; and the average annual unsupplied demand after outage $F_{R}$ indicates the reliability. Then the comprehensive objective is formulated as:

$$
\left\{\begin{array}{l}
F_{\mathrm{C}} \rightarrow \min \\
F_{\mathrm{T}} \rightarrow \max \\
F_{\mathrm{R}} \rightarrow \min
\end{array}\right.
$$

\subsection{Economic Cost}

The cost in the target year can be optimized by comparing a suitable set of alternative plans using the following economic model, which includes long-run investment and short-run operation cost for the substations, lines, CSs, and operation cost for the power loss:

$$
\begin{aligned}
& F_{\mathrm{C}}=C_{\text {sub }}+C_{\text {line }}+C_{\mathrm{FCS}}+C_{\text {loss }} \\
& C_{\text {sub }}=C_{\text {sub_new }}+C_{\text {sub_exp }}+C_{\text {sub_op }} \\
& =\frac{r_{0}\left(1+r_{0}\right)^{m_{\mathrm{S}}}}{\left(1+r_{0}\right)^{m_{\mathrm{S}}}-1}\left(\sum_{i \in \psi_{\mathrm{S}_{-} \mathrm{N}}} C_{i}^{\mathrm{S}_{-} \mathrm{N}} x_{i}^{\mathrm{S} \_\mathrm{N}}+\sum_{j \in \psi_{\mathrm{S}_{-} \mathrm{E}}} C_{j}^{\mathrm{S}_{-} \mathrm{E}} x_{j}^{\mathrm{S}-\mathrm{E}}\right)+D \delta^{\mathrm{S}} \sum_{t \in T} \sum_{k \in \psi_{\mathrm{S}}} P_{k, t}^{\mathrm{S}} x_{k}^{\mathrm{S}} \\
& C_{\text {line }}=C_{\text {line_new }}+C_{\text {line_maint }}=\frac{r_{0}\left(1+r_{0}\right)^{m_{\mathrm{L}}}}{\left(1+r_{0}\right)^{m_{\mathrm{L}}}-1} \sum_{(i j) \in \psi_{\mathrm{L} \_\mathrm{N}}} C^{\mathrm{L}} \mathrm{Len}_{i j} x_{i j}^{\mathrm{L} \_\mathrm{N}}+\delta^{\mathrm{L}} \sum_{(d p) \in \psi_{\mathrm{L}}} \operatorname{Len}_{d p} x_{d p}^{\mathrm{L}} \\
& C_{\mathrm{CS}}=\mathrm{C}_{\mathrm{CS}_{-} \text {fix }}+C_{\mathrm{CS}_{-} \text {var }}=\frac{r_{0}\left(1+r_{0}\right)^{m_{\mathrm{C}}}}{\left(1+r_{0}\right)^{m_{\mathrm{C}}}-1} \sum_{i \in \psi_{\mathrm{C}}} x_{i}^{\mathrm{CS}}\left(C_{i}^{\mathrm{CS} \_ \text {fix }}+s_{i} C_{i}^{\mathrm{CS} \_ \text {var }}\right)
\end{aligned}
$$




$$
C_{\text {loss }}=D \delta^{\operatorname{loss}} \sum_{t \in T} \sum_{(i j) \in \psi_{\mathrm{L}}} x_{i j}^{\mathrm{L}} g_{i j}\left(V_{i, t}^{2}+V_{j, t}^{2}-2 V_{i, t} V_{j, t} \cos \theta_{i j, t}\right)
$$

where $F_{\mathrm{c}}$ is the total cost in the target year, including four parts. $C_{\text {sub }}$ is the total cost of the substations, made up of the new installation investment $C_{\text {sub_new, }}$, the expansion investment $C_{\text {sub_exp }}$, and the operation cost $C_{\text {sub_op. }} C_{\text {line }}$ is the total cost for the lines, made up of the new installation investment $C_{\text {line_new }}$ and the operation and maintenance cost $C_{\text {line_maint. }} C_{\mathrm{CS}}$ is the total cost for the CSs, made up of the fixed investment $C_{C_{-} \text {fix }}$ and the variable investment $C_{C S}$ _var. $C_{\text {loss }}$ is the total cost of the power loss.

\subsection{CS Utilization}

The utilization of the CSs is reflected by the captured equilibrium traffic flow in the T-network, and the average annual captured traffic flow $F_{\mathrm{T}}$ is formulated as:

$$
F_{\mathrm{T}}=D \sum_{j \in \Omega_{\mathrm{T}}} \sum_{t \in T} f n_{j, t} x_{j}^{\mathrm{CS} \_\mathrm{T}}\left(x_{j *}^{\mathrm{CS}}\right)
$$

The proposed CS utilization index is used to describe the service capability of the candidate CS planning.

\subsection{Reliability Level}

The reliability level is presented to assess the power supply capability of the system dealing with faults from the view of structure connection, load demand, and others. It can quantify the impacts of the charging demand on the D-network besides the power balance or other network constraints, as well as the deployments of the generation units and the network reinforcement. The reliability is reflected by using the average annual unsupplied demand after outage, as given in the following equation:

$$
F_{\mathrm{R}}=D \sum_{t \in T} \sum_{m \in \psi_{\mathrm{a}}\left(x_{i}^{S}\right)} E N S_{m, t}
$$

The detailed connection conditions of a certain sub-area depend on the candidate plan. $E N S_{m, t}$ is the unsupplied power demand of sub-area $m$ in time period $t$ according to information including the failure rate, the repair time, etc. It is noted that the load transfer capability of the sub-area is determined by both the transmission capacity of the interconnection line and the generation margin of the sub-area that provides the load transfer in the time period $t$.

\subsection{Constraints}

(1) Power balance equations:

$$
\begin{gathered}
P_{i, t}^{\mathrm{S}} x_{i}^{\mathrm{S}}-P_{\mathrm{L} i, t}-P_{i, t}^{\mathrm{CP}}-P_{i, t}^{\mathrm{CS}} x_{i}^{\mathrm{CS}}=V_{i, t} \sum_{j \in N_{D}} V_{j, t}\left(G_{i j}\left(x_{i j}^{\mathrm{L}}\right) \cos \theta_{i j, t}+B_{i j}\left(x_{i j}^{\mathrm{L}}\right) \sin \theta_{i j, t}\right) \\
Q_{i, t}^{\mathrm{S}} x_{i}^{\mathrm{S}}-Q_{\mathrm{L} i, t}=V_{i, t} \sum_{j \in N_{D}} V_{j, t}\left(G_{i j}\left(x_{i j}^{\mathrm{L}}\right) \sin \theta_{i j, t}-B_{i j}\left(x_{i j}^{\mathrm{L}}\right) \cos \theta_{i j, t}\right)
\end{gathered}
$$

(2) Voltage magnitude:

$$
V^{\min } \leq V_{i, t} \leq V^{\max }
$$

(3) Power flow of the lines:

$$
\left|P_{i j}\right| \leq P_{i j}^{\max }
$$


(4) Power output for the substations:

$$
0 \leq P_{k, t}^{\mathrm{S}} \leq x_{k}^{\mathrm{S}}\left(P_{k}^{\mathrm{M} \_0} x_{k}^{\mathrm{S}-0}+P_{k}^{\mathrm{M} \_\mathrm{N}} x_{k}^{\mathrm{S} \_\mathrm{N}}+P_{k}^{\mathrm{M} \_\mathrm{E}} x_{k}^{\mathrm{S}-\mathrm{E}}\right)
$$

(5) The number of charging devices:

$$
s^{\min } x_{i}^{\mathrm{CS}} \leq s_{i} x_{i}^{\mathrm{CS}} \leq s^{\max } x_{i}^{\mathrm{CS}}
$$

(6) The geographical distance between any CS pair:

$$
d_{m-n} \geq d^{\min }
$$

where $d_{m-n}=\xi \sqrt{\left(X_{m}-X_{n}\right)^{2}+\left(Y_{m}-Y_{n}\right)^{2}}$, while $X_{m}$ and $Y_{m}$ represent the horizontal and vertical axis, respectively, of node $m$ in T-network, and $\xi$ is the distance modification coefficient [30].

(7) Radial topology in operation:

$$
n_{L}=n_{V}-n_{S}\left(x_{i}^{\mathrm{S}}\right)
$$

Equations (17), (18), and (24) constitute the radiality constraints.

It is worth noting that some other constraints during the calculation of charging demand, such as the traffic flow constraints of the transportation network and the consumer waiting time constraint for charging in CS, are integrated in the transportation network framework described in Section 3 and [17] as well.

\section{Multi-Stage Search Strategy}

According to the models in the previous sections, a multi-stage search strategy is designed for solving the ODNEP problem, as shown in Figure 4.

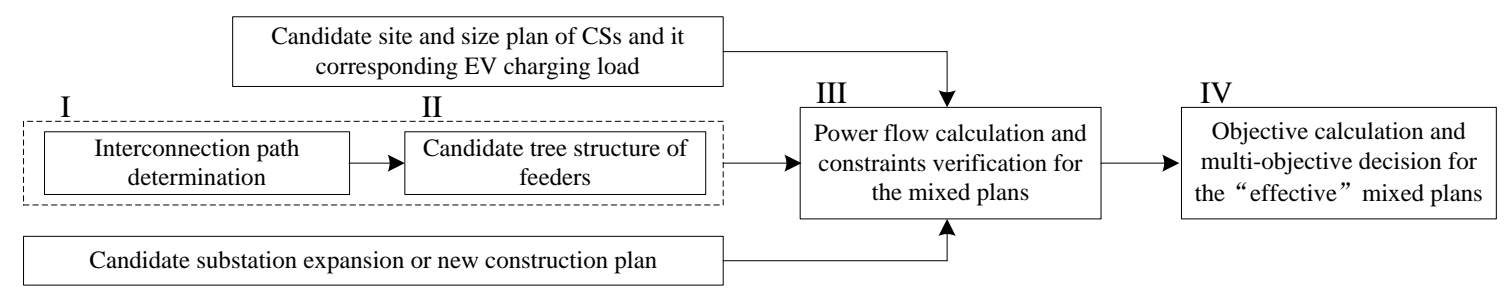

Figure 4. Multi-stage search strategy.

Stages I-II are used to generate the candidate network structure (topology). The interconnection paths should be determined in the first place according to the connection between the candidate substations, as shown in Stage I. According to the original topology and candidate substations information, the Floyd-Warshall algorithm [31] is used to find the shortest path with the minimum sum of line impedances between each generation bus (site of the substation). The reason for this is that, generally, the larger the impedance of a line is, the greater the investment; also, the power loss will be greater a larger current passes through the line. So, taking the shortest path can effectively reduce the investment and power loss. In that way, the lines in the paths can be included in the final plans as line set $\psi_{\mathrm{FW}}$.

After the interconnection paths are determined, at least one line would normally be open in real operation to connect the four tree structure sub-areas. In Stage II, to avoid loops in each sub-area, a modified Prim algorithm [32] is used to generate the possible topology for the distribution network. Three rules need to be addressed in the random generating based on the Prim algorithm: (1) The constraint in Equation (24) needs to be satisfied; (2) each generation bus (substation) should 
connect with one load bus at least; (3) in the real operation of the distribution network with multiple substations, one line along the path between each generation pair in set $\psi_{\mathrm{FW}}$ would be open, which is defined as the normal open interconnection line. Repeat the algorithm to generate a number of candidate network structure plans.

Figure 5 shows simple examples of the proposed topology generation algorithm. Bus 1 and Bus 5 are generation buses, while the rest are load buses. The number in the bracket is the weight of the corresponding line. For example, the weight of line 1-4 is 1 . So, according to the shortest path algorithm, line 1-4 and line 4-5 are selected into $\psi_{\mathrm{FW}}$, as shown in Figure 5a. Then, based on the designed three rules, the final candidate topology plans can be seen in Figure 5b-e.

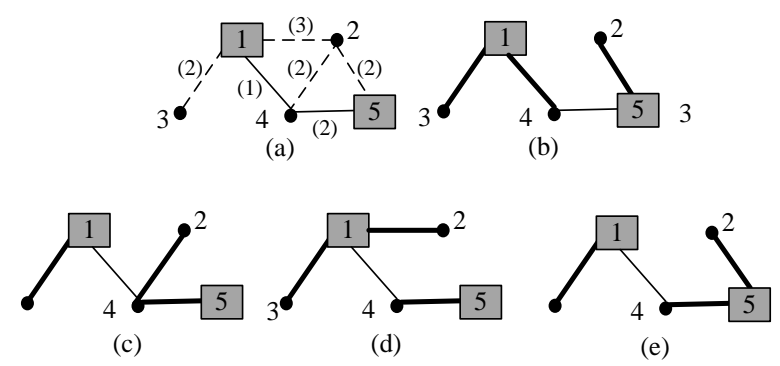

Figure 5. Examples of the proposed topology generation algorithm.

In Stage III, the candidate network structure plans, candidate substation plans, and candidate CS plans are combined to obtain "mixed" combination plans. Then power flow calculation and constraints verification would be implemented. If it failed to pass the verification, then the plan would be abandoned. Otherwise, the "effective" combination plans would be filtered. Then the performance (objectives) corresponding to each "effective" plan would be calculated. In terms of the calculation for the reliability level objective, the bus-bus connections matrix, interconnection line, and load transfer capability between each substations and other information should be cleared up first according to the network structure data in each effective plan, the label for each bus in each sub-area should be re-numbered, and a feeder matrix will be prepared for the ENS calculation based on the analytical method [33].

In Stage IV, a multi-objective decision would be implemented for each effective combination plan. Each objective has its own expected value and optimal trend. In terms of the minimum problem, the minimum value of $F(x)$ is called the best value, which is shown as $F\left(x^{*}\right)$ where $x^{*}$ is the optimal solution. Conversely, the worst value of $F(x)$ is the maximum value, defined as $F^{\mathrm{W}}$. Then the normalization is formulated as shown in Equation (25), which gives 0 as the minimum value and 1 as the maximum value:

$$
F_{i, j}^{\mathrm{N}}(x)=\frac{F_{i, j}(x)-F_{i}\left(x^{*}\right)}{F_{i}^{\mathrm{W}}-F_{i}\left(x^{*}\right)}
$$

where $F_{i, j}(x)$ is the value of the $i$ th objective function in the $j$ th candidate plan.

For the purpose of comparing the relative efficiencies of the various multi-objectives, a super criterion also known as the bargaining function is applied and formulated:

$$
\operatorname{Max}_{B} I_{j}=\prod_{i=1}^{R}\left(1-F_{i, j}^{N}(x)\right)^{\tau_{i}} j=1, \ldots, N
$$

where $N$ is the number of the candidate plans and $R$ is the number of the objectives, e.g., $R=3$ in the designed model of this paper. $B I_{j}(x)$ is the bargaining function, which is used to describe the distance from the solution point to the one with the worst value of all the objectives. The larger $B I_{j}$ is, meaning the further the distance is, the better the comprehensive performance of the corresponding solution will be. 
Finally, the optimal plan can be determined by selecting the one from the frontiers with the largest bargaining criterion, according to Equation (26).

\section{Test Cases}

\subsection{Case Description}

Sioux-Falls network [34] is used as the T-network in the test case, which contains 24 nodes, as shown in Figure 6. Bi-direction roads connect two neighboring nodes. Detailed daily trip 552 OD pairs traffic data can be found in [34]. A 54-bus distribution network is used as the D-network, as shown in Figure 6. It is a 15-kV system; the detailed systems data can be found in $[19,35]$. The solid lines are existing ones, while the dotted line are candidates. The "node-bus" pair of the coupled network can also be seen in Figure 6. The bus in the shadow space belongs to the commercial area while the others belong to residential area. The typical normalized daily parking demand, typical normalized daily load demand, and typical normalized daily traffic flow coefficient for planning $[16,26,28]$ are given in Figure 7. The goal of this test is planning a distribution system with four substations, two existing substations (one can be expanded), two candidate new substations, 5 CSs (11 candidate locations), and the line reinforcement from the candidate ones. Link lengths of the road network are set and scaled by $10^{-4}(\mathrm{~km})$ based on the given node coordinates [34]. Programs are implemented in the MATLAB environment using Celeron E3300 2.5 GHz/1.96 GB computers.
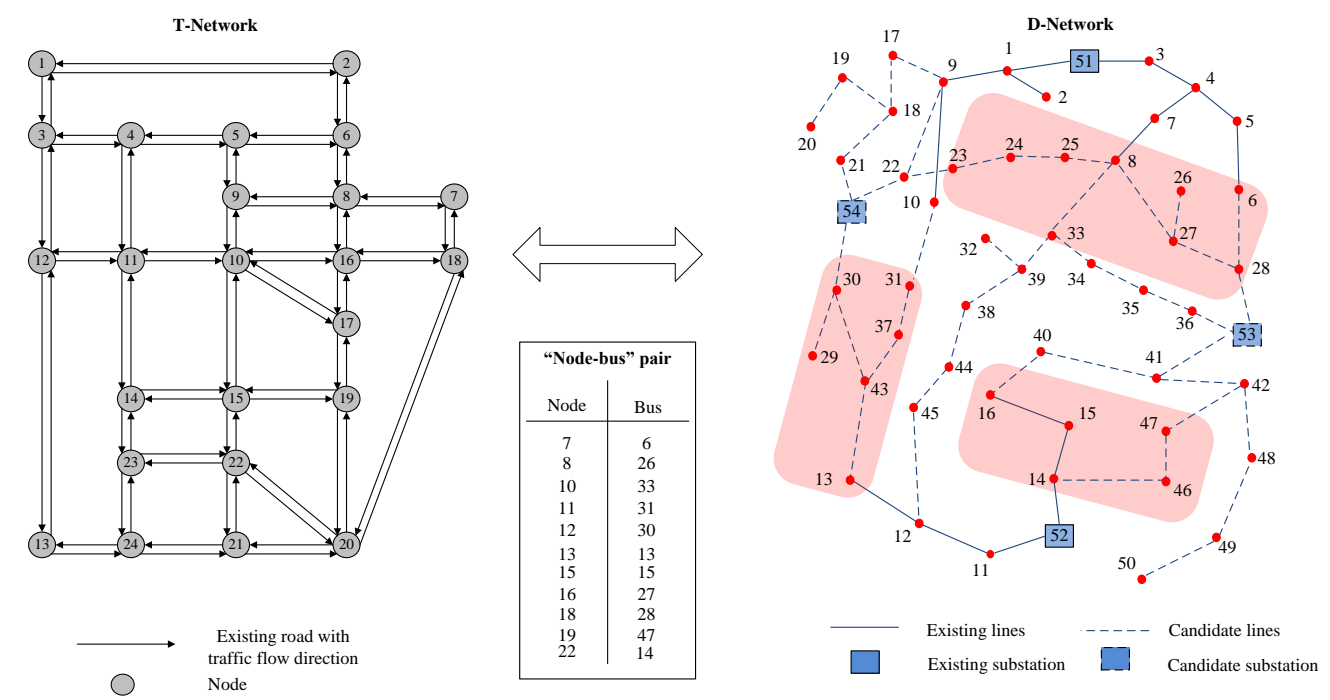

Figure 6. Test networks.

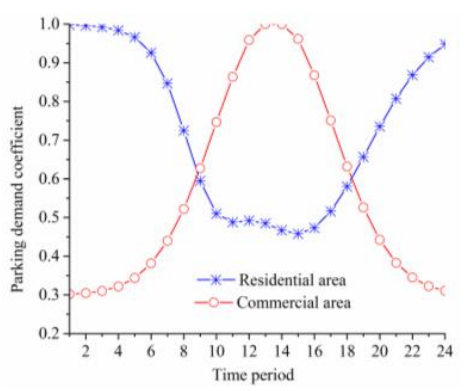

(a)

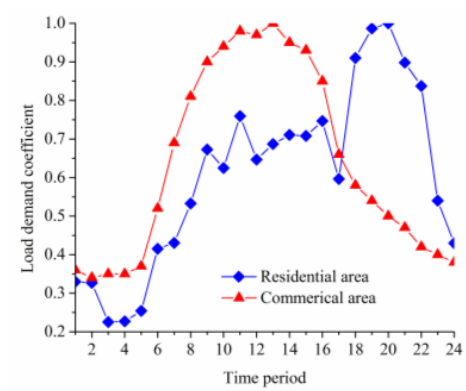

(b)

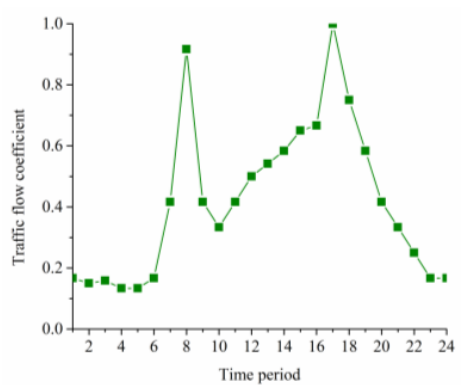

(c)

Figure 7. Typical normalized time curve. (a) Typical normalized daily parking demand time curve; (b) Typical normalized daily load demand time curve; (c) Typical normalized daily traffic flow coefficient time curve. 
The main parameters for the planning framework are given based on the real data and also the related settings in some studies, such as [17,19,36-38], which are set as follows for the case study: Let $H=3600, r_{0}=0.1, b=0.15, m_{S}=m_{L}=m_{C}=15, d^{\min }=10 \mathrm{~km}, \mu=0.5, W_{q}^{\max }=10 \mathrm{~min}, \omega=0.2$, $\omega=1, P_{\mathrm{CP}}=3 \mathrm{~kW}, P_{\mathrm{CD}}=30 \mathrm{~kW}, \gamma=0.75, \kappa=4$ vehicles $/$ day, $\mathrm{s}^{\text {min }}=4, \mathrm{~s}^{\text {max }}=10 . C^{L}=1 \times 10^{5} \mathrm{USD} / \mathrm{km}$, $\delta^{\text {loss }}=60 \mathrm{USD} / \mathrm{MWh}, \delta^{\mathrm{S}}=60 \mathrm{USD} / \mathrm{MWh}$, and $\delta^{\mathrm{L}}=1000 \mathrm{USD} / \mathrm{km}$ per year. The voltage magnitude varies within $[0.95,1.05]$ p.u. The transmission power limit for each line is set as $10 \mathrm{MW}$. The rest of the unit cost information for substations and CSs can be found in Tables 1 and 2.

Table 1. Substation expansion or new construction options.

\begin{tabular}{ccc}
\hline Station Location & Capacity Options (MW) & Investment $\left(\times \mathbf{1 0}^{\mathbf{6}} \mathbf{\text { USD}}\right)$ \\
\hline \multirow{2}{*}{ Bus 52 (expansion) } & 0 & 0 \\
& 5 & 8 \\
\hline \multirow{2}{*}{ Bus 53 (new) } & 5 & 7 \\
& 10 & 14 \\
& 15 & 21 \\
\hline \multirow{3}{*}{ Bus 54 (new) } & 5 & 6 \\
& 10 & 12 \\
\hline
\end{tabular}

Table 2. Unit fixed and variable investment for candidate CS $\left(\times 10^{4} \mathrm{USD}\right)$.

\begin{tabular}{cccccccccccc}
\hline Bus & $\mathbf{6}$ & $\mathbf{2 6}$ & $\mathbf{3 3}$ & $\mathbf{3 1}$ & $\mathbf{3 0}$ & $\mathbf{1 3}$ & $\mathbf{1 5}$ & $\mathbf{2 7}$ & $\mathbf{2 8}$ & $\mathbf{4 7}$ & $\mathbf{1 4}$ \\
\hline$C_{i}^{\mathrm{CS} \text { ffix }}$ & 35 & 27 & 45 & 38 & 25 & 20 & 40 & 45 & 45 & 35 & 35 \\
$C_{i}^{\mathrm{CS} \_v a r}$ & 11.5 & 10.7 & 12.5 & 11.8 & 10.5 & 10 & 12 & 12.5 & 12.5 & 11.5 & 12.5 \\
\hline
\end{tabular}

\subsection{Simulation Result and Analysis}

Based on the OD analysis and assignment method in [17], 19 available plans (sites and sizes) for the CSs can be obtained in the T-network. In each candidate CS plan, the corresponding hourly EV charging load profile can also be estimated and prepared for the coordinated planning.

Then, the interconnection paths between each generation bus (substation) pair are determined based on the proposed strategy in Stage I, as shown in Table 3.

Table 3. Lines in set $\psi_{\mathrm{FW}}$.

\begin{tabular}{cc}
\hline Station Pair (Bus) & Interconnection Path \\
\hline Bus 51-Bus 53 & Line 51-3, 3-4, 4-5, 5-6, 6-28, 28-53 \\
Bus 51-Bus 54 & Line 51-1, 1-9, 9-22, 22-54 \\
Bus 52-Bus 53 & Line 52-14, 14-15, 15-16, 16-40, 40-41, 41-53 \\
Bus 52-Bus 54 & Line 52-11, 11-12, 12-13, 13-43, 43-30, 30-54 \\
\hline
\end{tabular}

After that, several feasible network structure plans are generated by the designed method with its rules described in Stage II. Then, in the coupled networks, the "mixed" plans are formed and verified with multiple time period power flow calculation and constraints. Thus, there are 244 "effective" combination plans filtered, as scattered in Figure 8. In Figure 8, the feasible solution boundaries are formed as a cube, which includes the best and the worst value of each objective in the generated feasible solutions. When one objective achieves its best performance, there would be multiple combinations of the other two, but none are made up of the three best values of the objectives together. Let $\tau_{i}=1$, the bargaining criteria of each feasible solution after ranking as in Figure 9. According to the features of the bargaining decision, i.e., a larger value of the solution achieves better comprehensive performance, the best solution $(0.3637)$ can be chosen as the final optimal result. Its performance in terms of the 
three objectives is comprehensively best and has the furthest distance from the worst values. The final optimal planning result is as shown in Figure 10. The 50 lines in blue are deployed to guarantee the radial operation of the distribution network, and the dotted lines $22-54,6-28,16-40,13-43$ in red are also constructed as the normal open interconnection line for the generation of pairs of buses 51-54, 51-53, 52-53, and 52-54. The site and size of CSs can be also found in Figure 10, as well as the capacity result of the substations. It can be seen that the substations in Buses 51 and 52 do not need to be expanded, while $15 \mathrm{MW}$ substations need to be planned at Buses 53 and 54 .

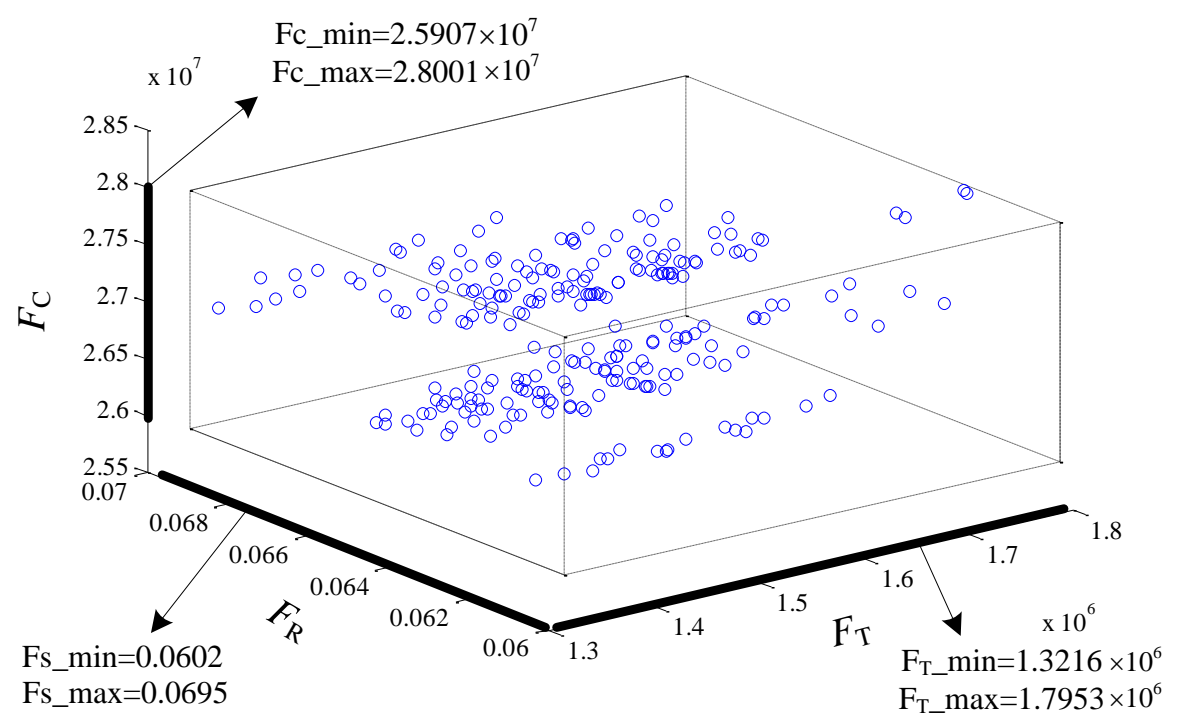

Figure 8. Multi-objective space of the effective plans.

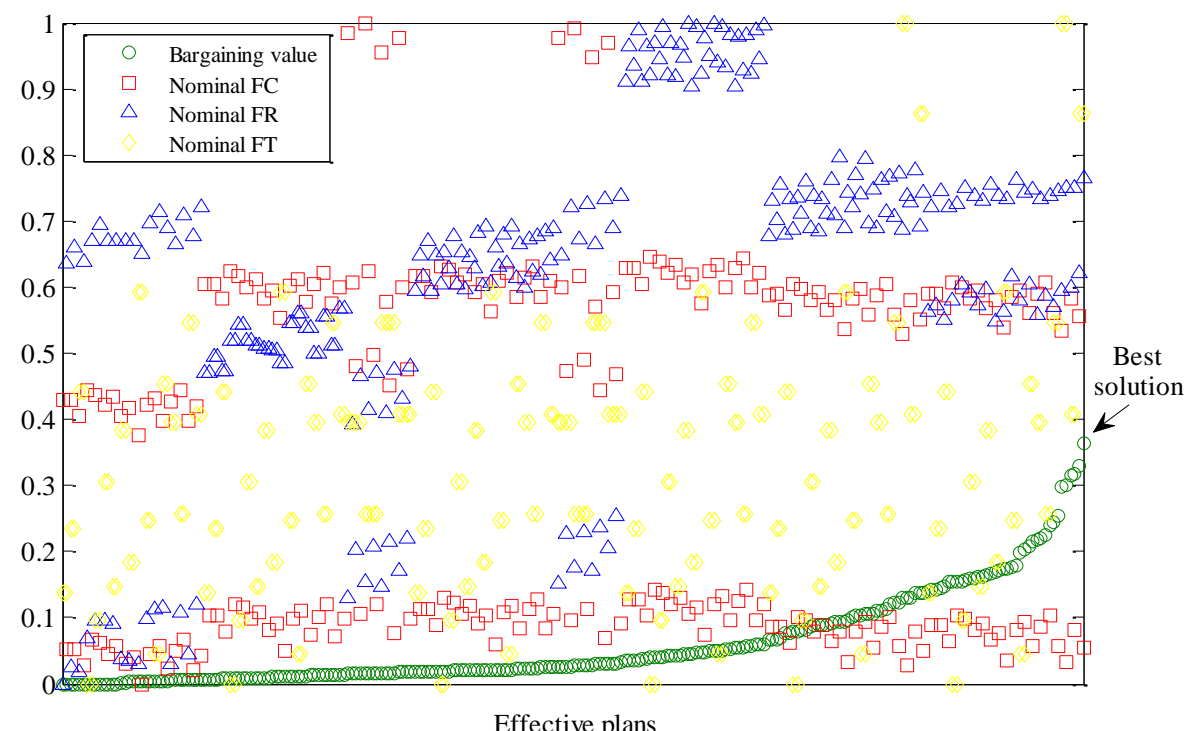

Figure 9. Bargaining function value and the normalized value of each objective. 


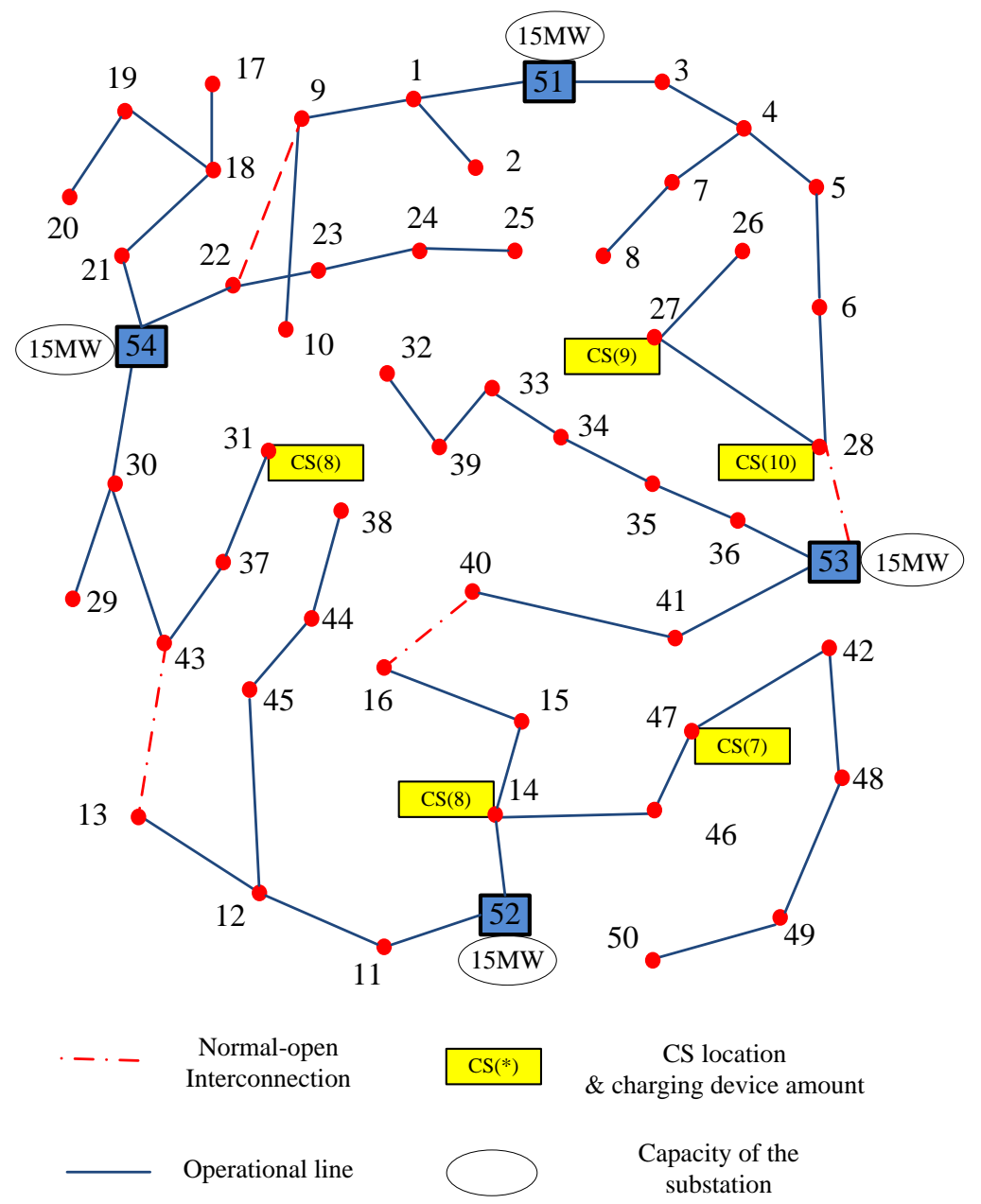

Figure 10. Optimal planning solution.

The results obtained with the proposed planning framework are compared with other results obtained through similar procedures reported in the literature, which are based on different objectives. The comparative results are shown in Table 4 . The result obtained based on the proposed planning framework in this paper is labeled as \#6. In the majority of prior studies, economic cost is the only concern, as in the method in [16]: $F_{\mathrm{C}}$ reaches the lowest in \#1 among all the effective combination plans, which is indeed the most economic one. However, if larger investment is permitted, the reliability of the plan stands at a comparative low level, as well as the ability to capture traffic flow. Thus the bargaining value of 0.0396 is comparatively small. Plan \#2 is obtained in view of the reliability of the system, and the coordination of "generation-network-load" is considered. The interactive impacts between the CS planning and distribution expansion are integrated into the reliability objective, but the other two objectives are not satisfied. The bargaining value of \#3 is close to that of \#6. However, the deployments for the network structure, CSs, and substations are quite different. The main reason is that $F_{\mathrm{T}}$ in \#3 purely considers the performance of the CS plan on the T-network, and $F_{\mathrm{C}}$ takes only the economic factors into consideration. The two integrated objectives cannot reflect the optimal solution to improve the performance of the couple networks besides power balance. However, in the multi-objective modeling of \#6, besides the two objectives, $F_{R}$ is introduced, which makes a comprehensive assessment on the "coupled" networks and deeply strengthens the meaning of "generation-network-load" coordination. The reliability objective is not a simple addition to the model, but is integrated into the algorithms and solving strategy including status analysis for the interconnection lines, distribution network partition, load transfer capability calculation, etc., which deepens the complexity compared with other studies, but gives better and more accurate 
planning results. The comparison between Plans \#5 and \#6 is similar to the discussion above. On the other hand, in terms of the proposed solution, it is simple, without multiply optimization iterations. Although the enumeration combination operation in the proposed multi-stage search strategy may generate a large number of "mixed" plans in the first place, the designed rule in Stage II of the proposed multi-stage search strategy can also help with dimension reduction. Besides, the network planning problem is not a real-time problem; there is enough time for optimization in the planning phase, and it is worth spending more time on optimization in the planning phase in order to get more accurate optimal solutions rather than "fast" ones.

Table 4. Planning results comparison with different modeling methods.

\begin{tabular}{|c|c|c|c|c|c|}
\hline \# & Description & $\begin{array}{c}F_{C} \\
\left(\times 10^{7} \$ / \text { Year }\right)\end{array}$ & $\begin{array}{c}F_{T} \\
\left(\times 10^{6} \text { Vehicles/Year }\right)\end{array}$ & $\begin{array}{c}F_{R} \\
(\mathrm{MWh} / \text { Year) }\end{array}$ & $\begin{array}{c}\text { Bargaining Value in the } \\
\text { Three-Objective } \\
\text { Optimization }\end{array}$ \\
\hline 1 & $F_{C} \rightarrow \min$ & 2.5907 & 1.4436 & 0.068 & 0.0396 \\
\hline 2 & $F_{R} \rightarrow \min$ & 2.7781 & 1.5935 & 0.0602 & 0.0403 \\
\hline 3 & $\begin{array}{l}F_{C} \rightarrow \min \\
F_{T} \rightarrow \max \end{array}$ & 2.688 & 1.7953 & 0.0639 & 0.3184 \\
\hline 4 & $\begin{array}{l}F_{C} \rightarrow \min \\
F_{R} \rightarrow \min \end{array}$ & 2.6662 & 1.3674 & 0.0607 & 0.0585 \\
\hline 5 & $\begin{array}{l}F_{T} \rightarrow \max \\
F_{R} \rightarrow \min \end{array}$ & 2.7932 & 1.7953 & 0.0625 & 0.0245 \\
\hline 6 & $\left\{\begin{array}{l}F_{C} \rightarrow \min \\
F_{T} \rightarrow \max \\
F_{R} \rightarrow \min \end{array}\right.$ & 2.6891 & 1.7953 & 0.0631 & 0.3637 \\
\hline
\end{tabular}

\section{Conclusions}

In this paper, a coordinated planning framework for distribution network expansion, including the siting and sizing of CSs, is proposed. Multi-objectives are designed in the optimal model, in which the minimization of long-run and short-run costs, the maximum of CS utilization, and the maximum of reliability are formulated from three perspectives. Moreover, a set of transportation and distribution operational constraints are included in the planning framework to capture EV travel behavior on the transportation network and charging effects on the distribution network. Furthermore, a multi-stage search strategy is presented on the geographically coupled transportation and distribution network to obtain the optimal planning solution.

The results of the test case are presented and compared with other modeling methods, which has shown the feasibility and effectiveness of the proposed model and strategy. In addition, it is shown that the multi-objective model can make a trade-off among minimization cost, maximization of CS utilization, and maximization of reliability, which is valuable for decision-makers. Also, the proposed distribution network expansion methodology can accommodate the integration of electric vehicles. Moreover, the presented framework can be used by local power companies to quantify the impacts of $\mathrm{EV}$ and the required expansion in the distribution network. Lastly, it also provides a way to evaluate the existing EV charging integrated network from the transportation and distribution perspectives.

Acknowledgments: This work was supported in part by the National Science Foundation of China (51377111) and in part by the Scientific Foundation for the Talent Program of Sichuan University (1082204112089) "Optimal allocation and integrated operation of power system considering widely integration of electric vehicles".

Author Contributions: This work is done by Yue Xiang under the supervision of Junyong Liu and Furong Li, and Wei Yang helped revise the paper.

Conflicts of Interest: The authors declare no conflict of interest. 


\section{Abbreviations}

The following abbreviations are used in this manuscript:

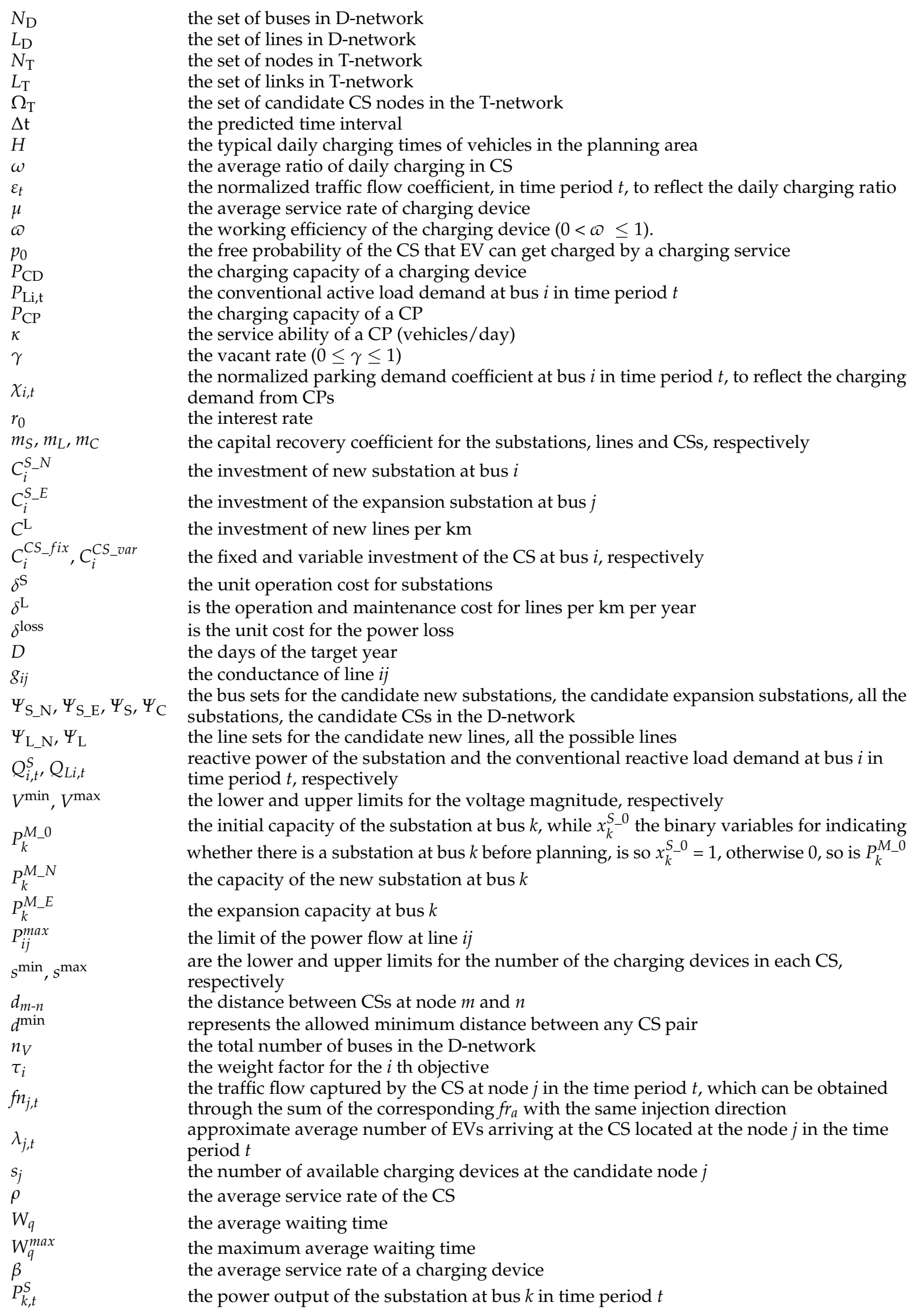




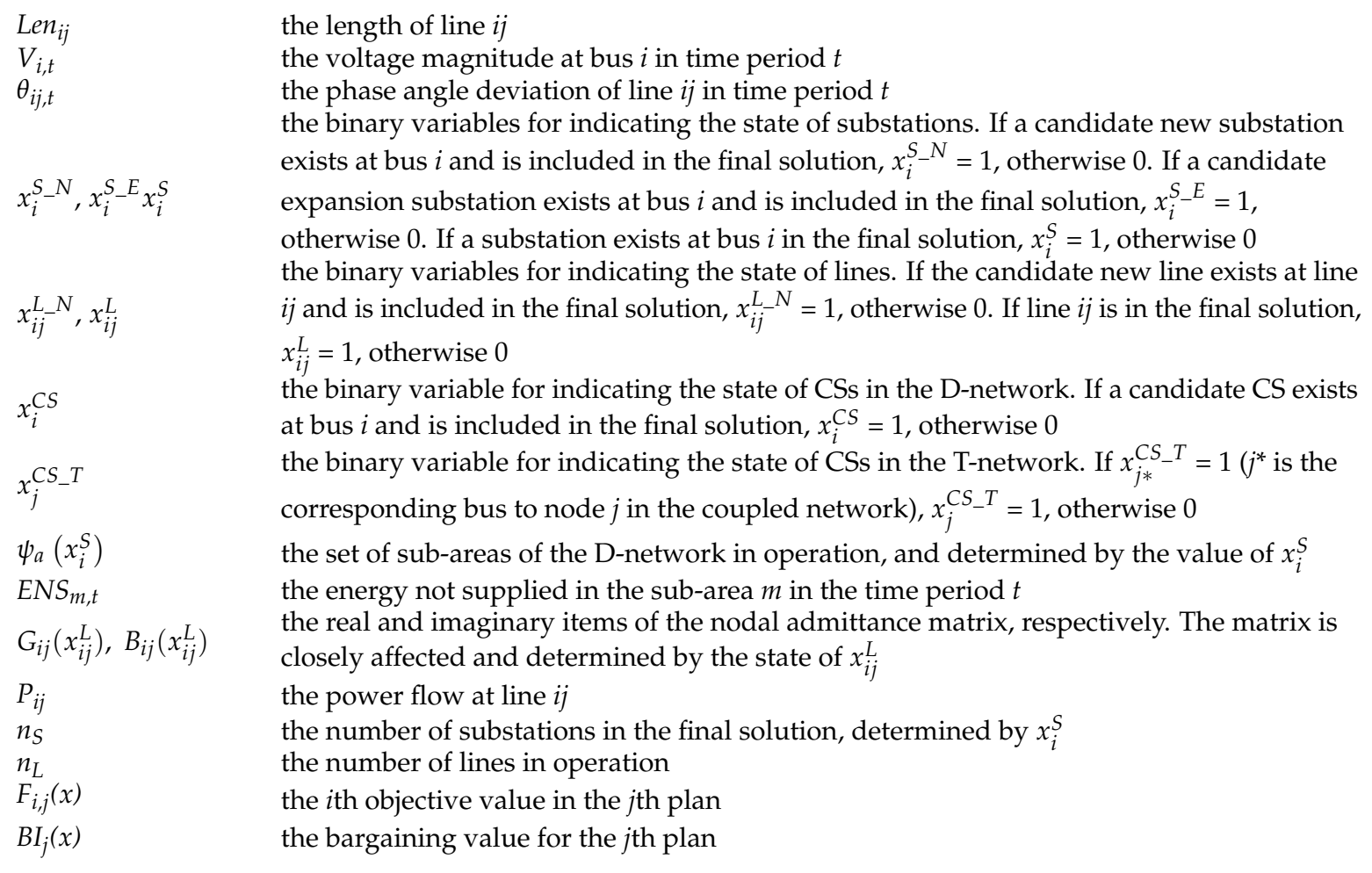

\section{References}

1. Zhou, L.; Li, F.; Gu, C.; Hu, Z.; le Blond, S. Cost/benefit assessment of a smart distribution system with intelligent electric vehicle charging. IEEE Trans. Smart Grid 2014, 5, 839-847. [CrossRef]

2. Xiang, Y.; Liu, J.; Li, F.; Liu, Y.; Liu, Y.; Xu, R.; Su, Y.; Ding, L. Optimal active distribution network planning: A review. Electr. Power Compon. Syst. 2016, 47, 1075-1094. [CrossRef]

3. Fernandez, L.P.; Roman, T.G.S.; Cossent, R.; Domingo, C.M.; Frias, P. Assessment of the impact of plug-in electric vehicles on distribution network. IEEE Trans. Power Syst. 2011, 26, 206-213. [CrossRef]

4. Shafiee, S.; Fotuhi-Firuzabad, M.; Rastegar, M. Investigating the impacts of plug-in hybrid electric vehicles on power distribution systems. IEEE Trans. Smart Grid 2013, 4, 1351-1360. [CrossRef]

5. Xiang, Y.; Liu, J.; Liu, Y. Optimal active distribution system management with aggregated plug-in electric vehicle. Electr. Power Syst. Res. 2016, 131, 105-115. [CrossRef]

6. He, F.; Yin, Y.; Lawphongpanich, S. Network equilibrium models with battery electric vehicles. Transp. Res. B 2014, 67, 306-319. [CrossRef]

7. Bayram, S.; Michalidis, G.; Devetsikiotics, M. Electric power allocation in a network of fast charging stations. IEEE J. Sel. Area Commun. 2013, 31, 1235-1246. [CrossRef]

8. Tang, X.; Liu, J.; Wang, X.; Xiong, J. Electric vehicle charging station planning based on computational geometry method. Autom. Electr. Power Syst. 2012, 36, 24-30.

9. Guo, S.; Zhao, H. Optimal site selection of electric vehicle charging station by using fuzzy TOPSIS based on sustainability perspective. Appl. Energy 2015, 158, 390-402. [CrossRef]

10. Liu, Z.; Wen, F.; Ledwich, G. Optimal planning of electric-vehicle charging stations in distribution systems. IEEE Trans. Power Deliv. 2013, 28, 102-110. [CrossRef]

11. Wang, Y.; Wang, C. Locating passenger vehicle refueling stations. Transp. Res. E Logist. Transp. Rev. 2010, 46, 791-801. [CrossRef]

12. Shukla, A.; Pekny, J.; Venkatasubramanian, V. An optimization framework for cost effective design of refueling station infrastructure for alternative fuel vehicles. Comput. Chem. Eng. 2011, 35, 1431-1438. [CrossRef]

13. Cavadas, J.; Correia, A.; Gouveia, G. A MIP model for locating slow-charging stations for electric vehicles in urban areas accounting for driver tours. Transp. Res. E Logist. Transp. Rev. 2015, 75, 188-201. [CrossRef]

14. Bayram, I.; Tajer, A.; Abdallah, M.; Qaraqe, K. Capacity planning frameworks for electric vehicle charging stations with multiclass customers. IEEE Trans. Smart Grid 2015, 6, 1924-1943. [CrossRef] 
15. Lin, W.; Hua, G. The flow capturing location model and algorithm of electric vehicle charging stations. In Proceedings of the 2015 International Conference on Logistics, Informatics, and Service Sciences, Beijing, China, 27-29 July 2015; pp. 27-29.

16. Xiang, Y.; Liu, J.; Tang, S.; Zhou, H. A traffic flow based planning strategy for optimal siting and sizing of charging stations. In Proceedings of the IEEE PES 2015 Asia-Pacific Power and Energy Conference, Brisbane, Australia, 15-18 November 2015; pp. 1-5.

17. Xiang, Y.; Liu, J.; Li, R.; Li, F.; Gu, C.; Tang, S. Economic planning of electric vehicle charging stations considering traffic constraints and load templates. Appl. Energy 2016, 178, 647-659. [CrossRef]

18. Wang, G.; Xu, Z.; Wen, F.; Wong, K.P. Traffic-constrained multi objective planning of electric-vehicle charging stations. IEEE Trans. Power Deliv. 2013, 28, 2363-2372. [CrossRef]

19. Lavorato, M.; Franco, J.F.; Rider, M.J.; Romero, R. Imposing radiality constraints in distribution system optimization problems. IEEE Trans. Power Syst. 2012, 27, 172-180. [CrossRef]

20. Ravadanegh, S.; Roshanagh, R. On optimal multistage electric power distribution networks expansion planning. Int. J. Electr. Power Energy Syst. 2014, 54, 487-497. [CrossRef]

21. Gao, C.; Zhang, L.; Xue, F.; Liu, H. Grid planning considering capacity and site of large-scale centralized charging stations. Proc. CSEE 2012, 32, 40-46.

22. Hu, Z.; Song, Y. Distribution network expansion planning with optimal siting and sizing of electric vehicle charging stations. In Proceedings of the 47th International Universities Power Engineering Conference, London, UK, 4-7 September 2012; pp. 1-6.

23. Lin, X.; Sun, J.; Ai, S.; Xiong, X.; Wan, Y.; Yang, D. Distribution network planning integrating charging stations of electric vehicle with V2G. Int. J. Electr. Power Energy Syst. 2014, 63, 507-512. [CrossRef]

24. Shaaban, M.; El-Saadany, E. Accommodating high penetrations of PEVs and renewable DG considering uncertainties in distribution systems. IEEE Trans. Power Syst. 2014, 29, 259-270. [CrossRef]

25. Zhang, Z. Study on Load Classification in Power System. Master's Thesis, Tianjin University, Tianjin, China, 2007.

26. Zhang, H.; Hu, Z.; Song, Y.; Xu, Z.; Jia, L. A prediction method for electric vehicle charging load considering spatial and temporal distribution. Autom. Electr. Power Syst. 2014, 38, 13-20.

27. Yang, W.; Zhou, H.; Liu, J.; Dai, S.; Ma, Z.; Liu, Y. Market evolution modeling for electric vehicles based on system dynamics and multi-Agents. In Proceedings of the 2015 International Symposium on Smart Electric Distribution Systems and Technologies (EDST), Wien, Austria, 8-11 September 2015.

28. Jia, L.; Hu, Z.; Liang, W.; Lang, W.; Song, Y. A novel approach for urban electric vehicle charging facility planning considering combination of slow and fast charging. In Proceedings of the 2014 International Conference on Power System Technology, Chengdu, China, 20-22 October 2014.

29. Li, R.; Su, H. Optimal allocation of charging facilities for electric vehicles based on queuing theory. Autom. Electr. Power Syst. 2011, 35, 58-61.

30. Feng, S.; Gao, H.; Guo, C. Evaluation of structural types of urban road network. J. Harbin Inst. Technol. 2007, $39,1610-1613$.

31. Cormen, T.; Leiserson, C. Introduction to Algorithms, 3rd ed.; China Machine Press: Beijing, China, 2012.

32. Liu, J.; Yang, W. Distribution networks expansion planning based on improved minimum-cost spanning tree. Autom. Electr. Power Syst. 2005, 29, 34-39.

33. Allan, R.N.; Billinton, R.; Sjarief, I.; Goel, L.; So, K.S. A reliability test system for education purposes: Basic distribution system data and results. IEEE Trans. Power Syst. 1991, 6, 813-820. [CrossRef]

34. Sioux-Falls Network. Available online: http://www.bgu.ac.il/ bargera/tntp/ (accessed on 31 August 2016).

35. Cui, H. Distribution Network Planning Based on Yearly Load Duration Curve. Mater's Thesis, North China Electric Power University, Beijing, China, 2011.

36. Zhang, S.; Li, K.; Cheng, H. Siting and sizing planning of distributed wind generators under active management mode. Autom. Electr. Power Syst. 2015, 39, 208-214.

37. Du, A.; Hu, Z.; Song, Y.; Wu, J. Distribution network planning considering layout optimization of electric vehicle charging stations. Power Syst. Technol. 2011, 35, 35-42.

38. Xiang, Y. Planning and Operation of Distribution System Considering Distributed Generation and Electric Vehicles. Ph.D. Thesis, Sichuan University, Chengdu, China, 2016.

(C) 2016 by the authors; licensee MDPI, Basel, Switzerland. This article is an open access article distributed under the terms and conditions of the Creative Commons Attribution (CC-BY) license (http://creativecommons.org/licenses/by/4.0/). 